\title{
Impact of Ventilatory Disorders on Respiratory Symptoms, Physical Activity, and Quality of Life in Post-Tuberculosis Subjects: A National Database Study in Korea
}

\section{Bumhee Yang}

Chungbuk National University Hospital

Hayoung Choi

Hallym University Sacred Heart Hospital

\section{Sun Hye Shin}

Samsung Medical Center

\section{Youlim Kim}

Hallim Daehakgyo Chuncheon Seongsim Byeongwon: Chuncheon Sacred Heart Hospital

\section{Ji-Yong Moon}

Hanyang University Seoul Medical Center: Hanyang University Seoul Hospital

Hye Yun Park

Samsung Medical Center

Hyun Lee ( $\square$ namuhanayeyo@naver.com )

Department of Internal Medicine, Hanyang University College of Medicine https://orcid.org/0000-0002$1269-0913$

\section{Research}

Keywords: tuberculosis, pulmonary function, respiratory symptoms, quality of life

Posted Date: June 15th, 2021

DOl: https://doi.org/10.21203/rs.3.rs-567154/v1

License: (9) This work is licensed under a Creative Commons Attribution 4.0 International License. Read Full License 


\section{Abstract}

\section{Background}

Tuberculosis (TB) survivors experience post-TB lung damage and ventilatory function disorders. However, the proportions of obstructive and restrictive ventilatory disorders as well as normal ventilation among post-TB subjects are unknown. In addition, the impacts of ventilatory disorder and its severity on respiratory symptoms, physical activity limitations, and the quality of life in post-TB subjects remain unclear.

\section{Methods}

Subjects who participated in the Korean National Health and Nutritional Examination Survey 2007-2016 were enrolled in this study. We evaluated the impact of each ventilatory disorder and its severity on respiratory symptoms, physical activity limitations, and quality of life (measured by the EuroQoL five dimensions questionnaire [EQ-5D] index values) in post-TB subjects.

Results

Among 1,466 post-TB subjects, $29 \%$ and $16 \%$ had obstructive ventilatory disorders and restrictive ventilatory disorders, respectively. Mild and moderate obstructive ventilatory disorders were not associated with respiratory symptoms, physical activity limitation, or EQ-5D index value compared with normal ventilation; however, severe obstructive ventilatory disorders were associated with more respiratory symptoms (adjusted odds ratio $[\mathrm{aOR}]=5.82,95 \%$ confidence interval $[\mathrm{Cl}]=2.80-12.10$ ), more physical activity limitation ( $\mathrm{aOR}=92.20,95 \% \mathrm{Cl}=16.33-520.62)$, and decreased EQ-5D index (adjusted coefficient $=-0.055,95 \% \mathrm{Cl}=-0.096--0.013)$ compared with normal ventilation. Mild restrictive ventilatory disorders were associated with more respiratory symptoms $(\mathrm{aOR}=1.95,95 \% \mathrm{Cl}=1.07-3.56)$ compared with normal ventilation, while moderate $(\mathrm{aOR}=9.17,95 \% \mathrm{Cl}=1.02-82.22)$ and severe restrictive symptoms $(\mathrm{aOR}=9.17,95 \% \mathrm{Cl}=1.02-82.22)$ were associated with physical activity limitation compared with normal ventilation.

Conclusion

Among post-TB subjects, $29 \%$ and $16 \%$ developed obstructive and restrictive ventilatory disorders, respectively. Severe obstructive ventilatory disorder was associated with more respiratory symptoms, more physical activity limitation, and poorer quality of life, while severe restrictive ventilatory disorder was associated with more physical activity limitations.

\section{Background}

Tuberculosis (TB) is the leading cause of death from a single epidemic disease worldwide [1]. After recognition of TB as a deadly communicable disease, the mortality rate has decreased, and $85 \%$ of patients treated for the first episode of TB survive with treatment success (cure or completion).[1, 2] 
Despite the improved survival rate, approximately $50 \%$ of TB survivors experience post-TB lung damage and ventilatory disorders. [3-5] Depending on the severity of post-TB lung damage, post-TB subjects show various clinical courses, including no respiratory symptoms, dyspnea, or impaired quality of life (QoL).[6, 7]

The ventilatory disorders of post-TB subjects comprise obstructive and restrictive patterns.[8] Obstructive ventilatory disorder, which is airflow limitation on pulmonary function tests, is the most well-known form of lung damage after TB treatment.[5, 8] Post-TB lung damage, including cavity, bronchiectasis, or distorted airway, can cause obstructive ventilatory disorder, which can lead to dyspnea, chronic obstructive pulmonary disease (COPD), and reduced exercise capacity.[9, 10] Although obstructive disorder has been recognized as post-TB lung damage, restrictive ventilatory disorder also is found in post-TB subjects and can lead to dyspnea or chest pain.[11] The restrictive pattern has been suggested to be a consequence of excessive fibrosis, fibrotic bands, or bronchovascular distortion in the process of post-TB lung repair.[12] The proportions of obstructive and restrictive ventilatory disorders as well as normal ventilation among post-TB subjects are unknown. Additionally, the impacts of ventilatory defects and severity of defects on respiratory symptoms, physical limitations, and QoL in post-TB subjects have not been investigated.

This study aimed to elucidate the composition of ventilatory defects among post-TB survivors using a nationally representative database in South Korea. Furthermore, this study investigated the impact of each ventilatory disorder and its severity on respiratory symptoms, physical activity limitations, and QoL among post-TB subjects

\section{Methods}

\section{Study population}

We used the data from 2007-2016 from the Korea National Health and Nutrition Examination Survey (NHANES), a nationally representative health survey collected by the Korean Ministry of Health and Welfare. Health-related questionnaires, health examinations, and spirometry results were used in this study. Previous pulmonary TB was defined based on formal reading of a chest X-ray or a history of physician diagnosed pulmonary TB. We classified post-TB subjects into three groups according to spirometric pattern (Fig. 1). The study protocol was approved by the Institutional Review Board of Chungbuk National University Hospital (application no. 2021-01-041).

\section{Measurements}

Data on age, sex, body mass index (BMI), smoking history, physical activity limitations, occupation, EuroQoL five dimensions questionnaire (EQ-5D) index value, and spirometric results were obtained from the Korea NHANES database. The EQ-5D index, which is used to measure QoL, ranges between 0 (worst imaginable health state) and 1 (best imaginable health state). Spirometry was performed according to 
the recommendations of the American Thoracic Society and European Respiratory Society.[13] Absolute values of forced expiratory volume in 1 second $\left(\mathrm{FEV}_{1}\right)$ and forced vital capacity $(\mathrm{FVC})$ were obtained, and the percentage of predicted values (\% predicted) for $\mathrm{FEV}_{1}$ and $\mathrm{FVC}$ were calculated using the reference equation obtained on analysis of a representative Korean sample.[14] Comorbidities of asthma, diabetes mellitus, hypertension, dyslipidemia, cardiovascular disease, osteoporosis, osteoarthritis or rheumatoid arthritis, and depression were self-reported based on previous physician diagnosis.[15]

\section{Definitions of ventilatory disorder}

Normal ventilation was defined as pre-bronchodilator $\mathrm{FEV}_{1} / \mathrm{FVC} \geq 0.70$ and $\mathrm{FVC} \geq 80 \%$ predicted. Obstructive ventilatory disorder was defined as pre-bronchodilator $\mathrm{FEV}_{1} / \mathrm{FVC}<0.70$.[16] For cases with obstructive ventilatory disorder, $\mathrm{FEV}_{1} \geq 80 \%$ predicted, $\mathrm{FEV}_{1}$ of $50-79$ \%predicted, and $\mathrm{FEV}_{1}<50$ $\%$ predicted were classified as mild, moderate, and severe, respectively. Restrictive ventilatory disorder was defined as $\mathrm{FEV}_{1} / \mathrm{FVC} \geq 0.7$ and $\mathrm{FVC}<80 \%$ predicted. For cases with restrictive ventilatory disorder, $\mathrm{FVC} \geq$ 70 \%predicted, FVC of 60-69 \%predicted, and FVC $<60 \%$ predicted were classified as mild, moderate, and severe, respectively.[17, 18]

\section{Outcomes}

We compared respiratory symptoms, physical activity limitations due to respiratory diseases (hereafter physical activity limitations), and QoL (measured by EQ-5D index) between post-TB subjects with different ventilatory disorders. We also analyzed the impacts of the severity of the ventilatory disorder on respiratory symptoms, physical activity limitations, and QoL in post-TB subjects.

\section{Statistical analysis}

All analysis was performed using survey commands in STATA 15.1 version (StataCorp LP, College Station, TX, USA) to account for the complex sampling design and survey weights. For each variable, we calculated prevalence and $95 \%$ confidence interval $(\mathrm{Cl})$ by group.

The associations between ventilatory disorders and respiratory symptoms (cough, sputum, or dyspnea) and physical activity limitations were analyzed using logistic regression analysis: Model 1 was adjusted for age (categorized as $\geq 65$ years or not), sex, and BMl; Model 2 was additionally adjusted for education level (categorized as high school or less vs. college or above) and family income (categorized as low vs. high). A linear regression analysis was performed to assess the association between ventilatory disorders and the EQ-5D index scores: Model 1 and Model 2 were adjusted for covariates as mentioned above. We used trend tests to evaluate whether there was a trend between severity of ventilator disorder and study outcome.

All tests were two-sided, and $p$-values $<0.05$ were considered to indicate statistically significant differences.

\section{Results}




\section{Baseline characteristics}

As shown in Table 1, the post-TB patient group included 1,466 patients $(54.9 \%)$ with normal ventilation, 783 patients $(29.3 \%)$ with obstructive ventilatory disorders, and 420 patients $(15.8 \%)$ with restrictive ventilatory disorders. The mean ages of subjects with normal ventilation, obstructive ventilatory disorders, and restrictive ventilatory disorders were 53.4, 64.4, and 59.6 years, respectively $(P<0.001)$. The proportion of males was highest in subjects with obstructive ventilatory disorders, followed by those with restrictive ventilatory disorders and those with normal ventilation $(76.2 \%, 52.0 \%$, and $49.5 \%$, respectively, $P<0.001)$. Subjects with obstructive ventilatory disorders had lower $\mathrm{BMI}$ than those with normal ventilation or restrictive ventilatory disorders $\left(22.8 \mathrm{~kg} / \mathrm{m}^{2}, 23.7 \mathrm{~kg} / \mathrm{m}^{2}\right.$, and $23.8 \mathrm{~kg} / \mathrm{m}^{2}$, respectively, $P<0.001)$. The proportion of subjects with low family income was highest in subjects with obstructive ventilatory disorders, followed by those with restrictive ventilatory disorders and those with normal ventilation $(68.3 \%, 55.9 \%$, and $43.0 \%$, respectively, $P<0.001)$. The subjects with obstructive ventilatory disorders had the highest prevalence of asthma (10.9\%), diabetes mellitus (19.1\%), and hypertension (50.8\%) among post-TB subjects, while subjects with restrictive ventilatory disorders had the highest prevalence of dyslipidemia (48.7\%) and osteoporosis (11.9\%) among the study population. Regarding spirometric results, FVC (L) and FVC \%predicted were lowest in subjects with restrictive ventilatory disorders, while $\mathrm{FEV}_{1}(\mathrm{~L}), \mathrm{FEV}_{1}$ \%predicted, and $\mathrm{FEV}_{1} / \mathrm{FVC}$ were lowest in those with obstructive ventilatory disorders among post-TB subjects $(P<0.001$ for all). 
Table 1

Baseline characteristics of post-TB subjects by spirometric pattern

\begin{tabular}{|c|c|c|c|c|c|}
\hline & $\begin{array}{l}\text { Total } \\
(n=2,669)\end{array}$ & $\begin{array}{l}\text { Normal } \\
\text { ventilation } \\
(n=1,466)\end{array}$ & $\begin{array}{l}\text { Obstructive } \\
\text { ventilatory disorder } \\
(n=783)\end{array}$ & $\begin{array}{l}\text { Restrictive } \\
\text { ventilatory disorder } \\
(n=420)\end{array}$ & $\begin{array}{l}\mathrm{p} \\
\text { value }\end{array}$ \\
\hline Age, years & $\begin{array}{l}57.5 \\
(56.7- \\
58.3)\end{array}$ & $\begin{array}{l}53.4(52.5- \\
65.5)\end{array}$ & $64.4(63.2-65.5)$ & $59.6(57.7-61.4)$ & $\begin{array}{l}<.001 \\
0.001\end{array}$ \\
\hline Male & $\begin{array}{l}57.7 \\
(55.2- \\
60.2)\end{array}$ & $\begin{array}{l}49.5(45.9- \\
53.1)\end{array}$ & $76.2(72.1-79.8)$ & $52.0(45.2-58.8)$ & $\begin{array}{l}< \\
0.001\end{array}$ \\
\hline $\mathrm{BMI}, \mathrm{kg} / \mathrm{m}^{2}$ & $\begin{array}{l}23.4 \\
(23.3- \\
23.6)\end{array}$ & $\begin{array}{l}23.7(23.5- \\
23.9)\end{array}$ & $22.8(22.5-23.1)$ & $23.8(23.4-24.3)$ & $\begin{array}{l}< \\
0.001\end{array}$ \\
\hline $\begin{array}{l}\text { Smoking } \\
\text { history }\end{array}$ & & & & & $\begin{array}{l}<.001 \\
0.001\end{array}$ \\
\hline Never-smoker & $\begin{array}{l}48.2 \\
(45.6- \\
50.8)\end{array}$ & $\begin{array}{l}55.5(51.8- \\
59.2)\end{array}$ & $28.2(24.1-32.6)$ & $60.6(53.5-67.2)$ & \\
\hline $\begin{array}{l}\text { Current- or ex- } \\
\text { smoker }\end{array}$ & $\begin{array}{l}51.8 \\
(49.2- \\
54.4)\end{array}$ & $\begin{array}{l}44.5(40.8- \\
59.2)\end{array}$ & $71.8(67.4-75.9)$ & $39.4(32.8-46.5)$ & \\
\hline Family income & & & & & <. 001 \\
\hline Low & $\begin{array}{l}52.1 \\
(49.2- \\
55.0)\end{array}$ & $\begin{array}{l}43.0(39.2- \\
46.8)\end{array}$ & $68.3(63.4-72.8)$ & $55.9(49.0-62.6)$ & \\
\hline High & $\begin{array}{l}47.9 \\
(45.0- \\
50.8)\end{array}$ & $\begin{array}{l}57.0(53.2- \\
60.8)\end{array}$ & $31.7(27.2-36.6)$ & $44.1(37.4-51.0)$ & \\
\hline Education & & & & & $\begin{array}{l}<.001 \\
0.001\end{array}$ \\
\hline $\begin{array}{l}\text { High school or } \\
\text { less }\end{array}$ & $\begin{array}{l}79.1 \\
(76.7- \\
81.3)\end{array}$ & $\begin{array}{l}74.9(71.6- \\
78.0)\end{array}$ & $86.5(82.5-89.7)$ & $80.4(73.9-89.7)$ & \\
\hline $\begin{array}{l}\text { More than high } \\
\text { school }\end{array}$ & $\begin{array}{l}20.9 \\
(18.7- \\
23.3)\end{array}$ & $\begin{array}{l}25.1(22.0- \\
28.4)\end{array}$ & $13.5(10.1-17.5)$ & $19.6(14.4-26.1)$ & \\
\hline \multicolumn{6}{|l|}{ Comorbidities } \\
\hline Asthma & $\begin{array}{l}4.9(3.9- \\
6.1)\end{array}$ & $\begin{array}{l}2.3(1.4- \\
3.6)\end{array}$ & $10.9(8.3-14.1)$ & $2.8(1.4-5.3)$ & $\begin{array}{l}<.001 \\
0.001\end{array}$ \\
\hline
\end{tabular}




\begin{tabular}{|c|c|c|c|c|c|}
\hline & $\begin{array}{l}\text { Total } \\
(n=2,669)\end{array}$ & $\begin{array}{l}\text { Normal } \\
\text { ventilation } \\
(n=1,466)\end{array}$ & $\begin{array}{l}\text { Obstructive } \\
\text { ventilatory disorder } \\
(n=783)\end{array}$ & $\begin{array}{l}\text { Restrictive } \\
\text { ventilatory disorder } \\
(n=420)\end{array}$ & $\begin{array}{l}p \\
\text { value }\end{array}$ \\
\hline $\begin{array}{l}\text { Diabetes } \\
\text { mellitus }\end{array}$ & $\begin{array}{l}12.4 \\
(10.7- \\
14.3)\end{array}$ & $\begin{array}{l}8.3(6.5- \\
10.5)\end{array}$ & $19.1(15.5-23.4)$ & $15.0(10.9-20.2)$ & $\begin{array}{l}< \\
0.001\end{array}$ \\
\hline Hypertension & $\begin{array}{l}41.4 \\
(38.7- \\
44.1)\end{array}$ & $\begin{array}{l}35.0(31.7- \\
38.6)\end{array}$ & $50.8(45.7-55.8)$ & $47.2(40.4-54.1)$ & $\begin{array}{l}< \\
0.001\end{array}$ \\
\hline Dyslipidemia & $\begin{array}{l}44.1 \\
(41.4- \\
47.0)\end{array}$ & $\begin{array}{l}42.9(39.2- \\
46.7)\end{array}$ & $44.5(39.5-49.7)$ & $48.4(41.5-55.3)$ & 0.413 \\
\hline $\begin{array}{l}\text { Cardiovascular } \\
\text { disease }\end{array}$ & $\begin{array}{l}5.1(4.0- \\
6.5)\end{array}$ & $\begin{array}{l}4.5(3.3- \\
6.2)\end{array}$ & $5.3(3.5-7.9)$ & $7.3(4.1-12.6)$ & 0.287 \\
\hline Osteoporosis & $\begin{array}{l}8.2(6.5- \\
10.3)\end{array}$ & $\begin{array}{l}7.4(5.5- \\
10.3)\end{array}$ & $7.7(4.6-12.5)$ & $11.9(7.3-19.0)$ & 0.297 \\
\hline Arthritis & $\begin{array}{l}15.1 \\
(13.4- \\
16.9)\end{array}$ & $\begin{array}{l}15.4(12.4- \\
18.0)\end{array}$ & $12.9(10.3-16.0)$ & $18.3(13.9-23.8)$ & 0.140 \\
\hline Depression & $\begin{array}{l}4.5(3.5- \\
5.6)\end{array}$ & $\begin{array}{l}4.4(3.2- \\
6.1)\end{array}$ & $4.6(2.9-7.1)$ & $4.4(2.4-7.7)$ & 0.993 \\
\hline \multicolumn{6}{|l|}{ Spirometry } \\
\hline FVC, L & $\begin{array}{l}3.5(3.4- \\
3.5)\end{array}$ & $\begin{array}{l}3.7(3.6- \\
3.8)\end{array}$ & $3.4(3.3-3.5)$ & $2.7(2.6-2.8)$ & $\begin{array}{l}< \\
0.001\end{array}$ \\
\hline $\begin{array}{l}\text { FVC, \% } \\
\text { predicted }\end{array}$ & $\begin{array}{l}88.7 \\
(88.0- \\
89.4)\end{array}$ & $\begin{array}{l}94.4(93.6- \\
95.1)\end{array}$ & $85.5(84.1-86.9)$ & $72.6(71.5-73.7)$ & $\begin{array}{l}< \\
0.001\end{array}$ \\
\hline $\mathrm{FEV}_{1}, \mathrm{~L}$ & $\begin{array}{l}2.6(2.5- \\
2.6)\end{array}$ & $\begin{array}{l}2.9(2.8- \\
3.0)\end{array}$ & $2.0(2.0-2.1)$ & $2.1(2.0-2.2)$ & $\dot{0.001}$ \\
\hline $\begin{array}{l}\mathrm{FEV}_{1}, \% \\
\text { predicted }\end{array}$ & $\begin{array}{l}84.7 \\
(83.8- \\
85.5)\end{array}$ & $\begin{array}{l}94.4(93.6- \\
95.1)\end{array}$ & $70.8(69.3-72.4)$ & $74.3(73.1-75.6)$ & $\begin{array}{l}< \\
0.001\end{array}$ \\
\hline $\mathrm{FEV}_{1} / \mathrm{FVC}$ & $\begin{array}{l}73.3 \\
(72.8- \\
74.0)\end{array}$ & $\begin{array}{l}78.9(78.6- \\
79.3)\end{array}$ & $60.6(59.7-61.4)$ & $78.0(77.2-78.7)$ & $\begin{array}{l}< \\
0.001\end{array}$ \\
\hline
\end{tabular}

Data are presented as weighted mean ( $95 \%$ confidence interval) or weighted percentage $(95 \%$ confidence interval).

TB, tuberculosis; BMI, body mass index; FVC, forced vital capacity; FEV $_{1}$, forced expiratory volume in 1 second. 


\section{Comparison of symptoms, physical activity, and quality of life}

As shown in Table 2, respiratory symptoms including sputum $(18.2 \%, 15.1 \%$, and $5.4 \%$, respectively, $P=$ $0.004)$ and dyspnea $(3.8 \%, 2.4 \%$, and $0.9 \%$, respectively, $P<0.020)$ and physical activity limitations $(27.7 \%, 13.0 \%$, and $5.1 \%$, respectively, $P<0.001)$ were most frequently observed in subjects with obstructive ventilatory disorders, followed by those with restrictive ventilatory disorders, and those with normal ventilation. Cough was observed most frequently in subjects with obstructive ventilatory disorders followed by those with restrictive ventilatory disorders or normal ventilation $(11.6 \%, 5.4 \%$, and $5.1 \%$, respectively, $P<0.001)$. 
Table 2

Comparison of symptoms, physical activity, and quality of life according to spirometric pattern

\begin{tabular}{|c|c|c|c|c|c|}
\hline & $\begin{array}{l}\text { Total } \\
(n= \\
2,669)\end{array}$ & $\begin{array}{l}\text { Normal } \\
\text { ventilation } \\
(n=1,466)\end{array}$ & $\begin{array}{l}\text { Obstructive } \\
\text { ventilatory disorder } \\
(n=783)\end{array}$ & $\begin{array}{l}\text { Restrictive } \\
\text { ventilatory } \\
\text { disorder } \\
(n=420)\end{array}$ & $\begin{array}{l}\mathrm{p} \\
\text { value }\end{array}$ \\
\hline $\begin{array}{l}\text { Any respiratory } \\
\text { symptoms }\end{array}$ & $\begin{array}{l}16.4 \\
(14.0- \\
19.3)\end{array}$ & $\begin{array}{l}12.4(9.5- \\
16.1)\end{array}$ & $22.9(18.1-28.5)$ & $18.6(12.6-26.6)$ & 0.001 \\
\hline Cough & $\begin{array}{l}7.3(5.7- \\
9.2)\end{array}$ & $\begin{array}{l}5.4(3.5- \\
8.3)\end{array}$ & $11.6(8.5-15.8)$ & $5.1(2.8-9.3)$ & 0.004 \\
\hline Sputum & $\begin{array}{l}13.0 \\
(10.8- \\
15.5)\end{array}$ & $\begin{array}{l}9.5(7.0- \\
12.8)\end{array}$ & $18.2(13.9-23.5)$ & $15.1(9.5-23.2)$ & 0.004 \\
\hline Dyspnea & $\begin{array}{l}2.0(1.3- \\
3.1)\end{array}$ & $\begin{array}{l}0.9(0.3- \\
2.5)\end{array}$ & $3.8(2.1-6.7)$ & $2.4(0.9-5.9)$ & 0.020 \\
\hline $\begin{array}{l}\text { Physical activity } \\
\text { limitations }\end{array}$ & $\begin{array}{l}15.6 \\
(11.2- \\
21.2)\end{array}$ & $\begin{array}{l}5.1(2.3- \\
10.9)\end{array}$ & $27.7(19.3-38.0)$ & $13.0(6.2-28.4)$ & $\dot{0.001}$ \\
\hline \multicolumn{6}{|l|}{ EQ-5D component } \\
\hline Mobility & $\begin{array}{l}18.3 \\
(16.4- \\
20.3)\end{array}$ & $\begin{array}{l}14.0 \\
(11.9- \\
16.4)\end{array}$ & $24.1(20.4-28.1)$ & $23.5(18.1-29.8)$ & $\begin{array}{l}<.001 \\
0.00\end{array}$ \\
\hline Self-care & $\begin{array}{l}5.2(4.3- \\
6.6)\end{array}$ & $\begin{array}{l}3.6(2.6- \\
5.0)\end{array}$ & $7.7(5.5-10.7)$ & $7.3(4.3-12.4)$ & 0.005 \\
\hline Usual activity & $\begin{array}{l}12.8 \\
(11.1- \\
14.6)\end{array}$ & $\begin{array}{l}9.4(7.7- \\
11.5)\end{array}$ & $16.3(13.1-20.1)$ & $19.1(14.2-25.2)$ & $\begin{array}{l}<.001 \\
0.00\end{array}$ \\
\hline Pain/discomfort & $\begin{array}{l}29.0 \\
(26.6- \\
31.5)\end{array}$ & $\begin{array}{l}30.7 \\
(26.5- \\
35.2)\end{array}$ & $30.1(26.5-35.2)$ & $36.3(29.8-43.4)$ & 0.015 \\
\hline Anxiety/depression & $\begin{array}{l}13.6 \\
(11.9- \\
15.5)\end{array}$ & $\begin{array}{l}13.5 \\
(11.3- \\
16.1)\end{array}$ & $13.5(10.6-17.1)$ & $13.7(9.5-17.5)$ & 0.999 \\
\hline EQ-5D index & $\begin{array}{l}0.93 \\
(0.92- \\
0.93)\end{array}$ & $\begin{array}{l}0.94 \\
(0.93- \\
0.94)\end{array}$ & $0.91(0.90-0.93)$ & $0.91(0.89-0.93)$ & 0.002 \\
\hline
\end{tabular}

Data are presented as weighted mean ( $95 \%$ confidence interval) or weighted percentage ( $95 \%$ confidence interval).

EQ-5D, EuroQoL five dimensions. 
The EQ-5D index values, denoting QoL, were lower among subjects with obstructive ventilatory disorders and restrictive ventilatory disorders than in those with normal ventilation $(0.91,0.91$, and 0.94 , respectively, $P=0.002$ ). Regarding the individual EQ-5D component arm, subjects with obstructive ventilatory disorders had the highest rates of difficulty in mobility $(24.1 \%, P<0.001)$ and limitation in selfcare $(7.7 \%, P=0.005)$ among post-TB subjects; however, those with restrictive ventilatory disorders had the highest rates of difficulty in usual activity $(19.1 \%, P<0.001)$ and pain/discomfort $(36.3 \%, P=0.015)$ among post-TB subjects (Table 2).

\section{The impact of obstructive ventilatory disorder and its severity on respiratory symptoms, physical activity limitations, and EQ-5D index in post-TB subjects.}

As shown in Table 3, subjects with obstructive ventilatory disorders had $1.68(95 \% \mathrm{Cl}=1.05-2.68)$ times more likely to have any respiratory symptoms compared to those with normal ventilation in the fully adjusted; additionally, it was specifically significant for sputum (aOR $=1.72,95 \% \mathrm{Cl}=1.34-3.39)$ and dyspnea $(\mathrm{aOR}=4.41,95 \% \mathrm{Cl}=1.40-13.96)$. Likewise, subjects with obstructive ventilatory disorder had more physical activity limitations $(\mathrm{aOR}=8.13,95 \% \mathrm{Cl}=2.95-22.41)$ compared to those with normal ventilation. Although subjects with obstructive ventilatory disorders were more likely to have a lower EQ$5 \mathrm{D}$ index compared to those with normal ventilation in the unadjusted model (coefficient $=-0.021,95 \% \mathrm{Cl}$ $-0.036--0.069)$, this was not significant in the adjusted model. 
Table 3

The impact of severity of obstructive ventilatory disorder on EQ-5D index value in post-TB subjects.

\begin{tabular}{|c|c|c|c|c|c|c|}
\hline & \multirow[t]{2}{*}{ Model } & \multirow{2}{*}{$\begin{array}{l}\text { Normal } \\
(n= \\
1,466)\end{array}$} & \multicolumn{4}{|c|}{ Obstructive ventilatory disorder } \\
\hline & & & $\begin{array}{l}\text { Overall } \\
(n=783)\end{array}$ & $\begin{array}{l}\text { Mild } \\
(n=256)\end{array}$ & $\begin{array}{l}\text { Moderate } \\
(n=432)\end{array}$ & $\begin{array}{l}\text { Severe } \\
(n=95)\end{array}$ \\
\hline \multirow[t]{2}{*}{$\begin{array}{l}\text { Respiratory } \\
\text { symptoms }\end{array}$} & $\begin{array}{l}\text { Crude } \\
\text { model }\end{array}$ & Reference & $\begin{array}{l}2.09(1.37, \\
3.21)\end{array}$ & $\begin{array}{l}1.52(0.83 \\
2.81)\end{array}$ & $\begin{array}{l}1.67(1.03, \\
2.71)\end{array}$ & $\begin{array}{l}8.38(4.16 \\
16.88)\end{array}$ \\
\hline & $\begin{array}{l}\text { Adjusted } \\
\text { model }^{*}\end{array}$ & Reference & $\begin{array}{l}1.68(1.05 \\
2.68)\end{array}$ & $\begin{array}{l}0.96(0.49, \\
1.90)\end{array}$ & $\begin{array}{l}1.29(0.77 \\
2.16)\end{array}$ & $\begin{array}{l}5.82(2.80 \\
12.10)\end{array}$ \\
\hline \multirow[t]{2}{*}{ Cough } & $\begin{array}{l}\text { Crude } \\
\text { model }\end{array}$ & Reference & $\begin{array}{l}2.30(1.27, \\
4.17)\end{array}$ & $\begin{array}{l}2.45(1.08, \\
5.56)\end{array}$ & $\begin{array}{l}1.74(0.87 \\
3.46)\end{array}$ & $\begin{array}{l}4.99(2.04 \\
12.28)\end{array}$ \\
\hline & $\begin{array}{l}\text { Adjusted } \\
\text { model }^{*}\end{array}$ & Reference & $\begin{array}{l}1.92(0.99 \\
3.69)\end{array}$ & $\begin{array}{l}1.69(0.65, \\
4.43)\end{array}$ & $\begin{array}{l}1.48(0.71, \\
3.07)\end{array}$ & $\begin{array}{l}3.81(1.44 \\
10.09)\end{array}$ \\
\hline \multirow[t]{2}{*}{ Sputum } & $\begin{array}{l}\text { Crude } \\
\text { model }\end{array}$ & Reference & $\begin{array}{l}2.13(1.34 \\
3.39)\end{array}$ & $\begin{array}{l}1.71(0.91, \\
3.22)\end{array}$ & $\begin{array}{l}1.69 \\
2.84)\end{array}(1.01$, & $\begin{array}{l}6.84(3.24 \\
14.44)\end{array}$ \\
\hline & $\begin{array}{l}\text { Adjusted } \\
\text { model }^{*}\end{array}$ & Reference & $\begin{array}{l}1.72(1.04 \\
2.86)\end{array}$ & $\begin{array}{l}1.26(0.62 \\
2.56)\end{array}$ & $\begin{array}{l}1.27(0.72 \\
2.23)\end{array}$ & $\begin{array}{l}4.53(1.98 \\
10.38)\end{array}$ \\
\hline \multirow[t]{2}{*}{ Dyspnea } & $\begin{array}{l}\text { Crude } \\
\text { model }\end{array}$ & Reference & $\begin{array}{l}4.05(1.30 \\
12.62)\end{array}$ & $\begin{array}{l}1.95(0.43, \\
8.88)\end{array}$ & $\begin{array}{l}2.12(0.56, \\
7.98)\end{array}$ & $\begin{array}{l}21.58(5.51, \\
84.46)\end{array}$ \\
\hline & $\begin{array}{l}\text { Adjusted } \\
\text { model }^{*}\end{array}$ & Reference & $\begin{array}{l}4.41(1.40 \\
13.96)\end{array}$ & $\begin{array}{l}1.96(0.44, \\
8.69)\end{array}$ & $\begin{array}{l}2.03(0.53 \\
7.78)\end{array}$ & $\begin{array}{l}19.02(4.06, \\
89.07)\end{array}$ \\
\hline \multirow{2}{*}{$\begin{array}{l}\text { Physical } \\
\text { activity } \\
\text { limitations }\end{array}$} & $\begin{array}{l}\text { Crude } \\
\text { model }\end{array}$ & Reference & $\begin{array}{l}7.20(2.93, \\
17.66)\end{array}$ & $\begin{array}{l}3.55(0.81 \\
15.46)\end{array}$ & $\begin{array}{l}3.60(1.25 \\
10.38)\end{array}$ & $\begin{array}{l}38.35 \\
(10.80 \\
136.15)\end{array}$ \\
\hline & $\begin{array}{l}\text { Adjusted } \\
\text { model }^{*}\end{array}$ & Reference & $\begin{array}{l}8.13(2.95, \\
22.41)\end{array}$ & $\begin{array}{l}3.00(0.74 \\
12.11)\end{array}$ & $\begin{array}{l}4.13(0.97 \\
17.64)\end{array}$ & $\begin{array}{l}92.20 \\
(16.33 \\
520.62)\end{array}$ \\
\hline \multirow[t]{2}{*}{ EQ-5D index } & $\begin{array}{l}\text { Crude } \\
\text { model }\end{array}$ & Reference & $\begin{array}{l}-0.021(- \\
0.036,- \\
0.069)\end{array}$ & $\begin{array}{l}-0.006 \\
(-0.261 \\
0.125)\end{array}$ & $\begin{array}{l}-0.020 \\
(-0.038 \\
-0.001)\end{array}$ & $\begin{array}{l}-0.066 \\
(-0.108 \\
-0.024)\end{array}$ \\
\hline & $\begin{array}{l}\text { Adjusted } \\
\text { model }^{*}\end{array}$ & Reference & $\begin{array}{l}-0.001(- \\
0.015,0.014)\end{array}$ & $\begin{array}{l}0.015 \\
(-0.005 \\
0.035)\end{array}$ & $\begin{array}{l}-0.002 \\
(-0.020 \\
0.015)\end{array}$ & $\begin{array}{l}-0.055 \\
(-0.096 \\
-0.013)\end{array}$ \\
\hline
\end{tabular}

Data are presented as a ratio ( $95 \%$ confidence interval) or a difference estimate ( $95 \%$ confidence interval).

*Age, sex, education (categorized as $>$ high school or $\leq$ high school), and family income (categorized as low or high) were adjusted. 
EQ-5D, EuroQoL five dimensions; TB, tuberculosis.

In the analyses according to severity of obstructive ventilatory disorders, mild and moderate obstructive ventilatory disorders were not associated with more respiratory symptoms, more physical activity limitations, and lower EQ-5D index values in the unadjusted and adjusted models. However, severe obstructive ventilatory disorder was significantly associated with more respiratory symptoms $(a O R=5.82$, $95 \% \mathrm{Cl}=2.80-12.10)$, more physical activity limitations $(\mathrm{aOR}=92.20,95 \% \mathrm{Cl}=16.33-520.62)$, and lower EQ-5D index value (adjusted coefficient $=-0.055,95 \% \mathrm{Cl}=-0.096--0.013)$ (Table 3).

The impact of restrictive ventilatory disorder and its severity on respiratory symptoms, physical activity limitations, and EQ-5D index value in post-TB subjects

Restrictive ventilatory disorder was not significantly associated with increased respiratory symptoms (cough, sputum, or dyspnea), physical activity limitations, and EQ-5D index value compared with normal ventilation in adjusted models (Table 4). 
Table 4

The impact of severity of restrictive ventilatory disorder on EQ-5D index value in post-TB subjects

\begin{tabular}{|c|c|c|c|c|c|c|}
\hline & \multirow[t]{2}{*}{ Model } & \multirow{2}{*}{$\begin{array}{l}\text { Normal } \\
(n= \\
1,466)\end{array}$} & \multicolumn{4}{|c|}{ Restrictive ventilatory disorder } \\
\hline & & & $\begin{array}{l}\text { Overall } \\
(n=420)\end{array}$ & $\begin{array}{l}\text { Mild } \\
(n=306)\end{array}$ & $\begin{array}{l}\text { Moderate } \\
(n=77)\end{array}$ & $\begin{array}{l}\text { Severe } \\
(n=37)\end{array}$ \\
\hline \multirow[t]{2}{*}{$\begin{array}{l}\text { Respiratory } \\
\text { symptoms }\end{array}$} & $\begin{array}{l}\text { Crude } \\
\text { model }\end{array}$ & Reference & $\begin{array}{l}1.61(0.95 \\
2.72)\end{array}$ & $\begin{array}{l}1.95(1.09, \\
3.49)\end{array}$ & $\begin{array}{l}0.95(0.30 \\
3.06)\end{array}$ & $\begin{array}{l}0.59(0.11, \\
3.24)\end{array}$ \\
\hline & $\begin{array}{l}\text { Adjusted } \\
\text { model }^{\star}\end{array}$ & Reference & $\begin{array}{l}1.59(0.92 \\
2.77)\end{array}$ & $\begin{array}{l}1.95(1.07, \\
3.56)\end{array}$ & $\begin{array}{l}0.98(0.29 \\
3.36)\end{array}$ & $\begin{array}{l}0.56(0.09, \\
3.31)\end{array}$ \\
\hline \multirow[t]{2}{*}{ Cough } & $\begin{array}{l}\text { Crude } \\
\text { model }\end{array}$ & Reference & $\begin{array}{l}0.95(0.43 \\
4.17)\end{array}$ & $\begin{array}{l}0.96(0.39 \\
2.38)\end{array}$ & $\begin{array}{l}0.77(0.16 \\
3.80)\end{array}$ & $\begin{array}{l}1.11(0.14, \\
8.98)\end{array}$ \\
\hline & $\begin{array}{l}\text { Adjusted } \\
\text { model }^{\star}\end{array}$ & Reference & $\begin{array}{l}0.94(0.41 \\
2.13)\end{array}$ & $\begin{array}{l}0.95(0.39 \\
2.32)\end{array}$ & $\begin{array}{l}0.78(0.14, \\
4.32)\end{array}$ & $\begin{array}{l}1.06(0.12, \\
9.35)\end{array}$ \\
\hline \multirow[t]{2}{*}{ Sputum } & $\begin{array}{l}\text { Crude } \\
\text { model }\end{array}$ & Reference & $\begin{array}{l}1.70(0.93 \\
3.12)\end{array}$ & $\begin{array}{l}2.23(1.16 \\
4.26)\end{array}$ & $\begin{array}{l}0.42(0.09, \\
2.00)\end{array}$ & $\begin{array}{l}0.61(0.08, \\
4.77)\end{array}$ \\
\hline & $\begin{array}{l}\text { Adjusted } \\
\text { model }^{\star}\end{array}$ & Reference & $\begin{array}{l}1.71(0.91 \\
3.23)\end{array}$ & $\begin{array}{l}2.28(1.17 \\
4.49)\end{array}$ & $\begin{array}{l}0.45(0.09 \\
2.31)\end{array}$ & $\begin{array}{l}0.54(0.06, \\
4.91)\end{array}$ \\
\hline \multirow[t]{2}{*}{ Dyspnea } & $\begin{array}{l}\text { Crude } \\
\text { model }\end{array}$ & Reference & $\begin{array}{l}2.49(0.63, \\
9.81)\end{array}$ & $\begin{array}{l}\text { 1.20 (0.21, } \\
6.82)\end{array}$ & $\begin{array}{l}8.56(1.43 \\
51.34)\end{array}$ & $\begin{array}{l}1.94(0.20 \\
18.40)\end{array}$ \\
\hline & $\begin{array}{l}\text { Adjusted } \\
\text { model }^{\star}\end{array}$ & Reference & $\begin{array}{l}2.27(0.56 \\
9.27)\end{array}$ & $\begin{array}{l}0.80(0.11, \\
5.91)\end{array}$ & $\begin{array}{l}13.52(2.45 \\
74.82)\end{array}$ & $\begin{array}{l}2.83(0.24, \\
33.47)\end{array}$ \\
\hline \multirow{3}{*}{$\begin{array}{l}\text { Physical } \\
\text { activity } \\
\text { limitation }\end{array}$} & $\begin{array}{l}\text { Crude } \\
\text { model }\end{array}$ & Reference & $\begin{array}{l}2.98(0.76 \\
9.45)\end{array}$ & $\begin{array}{l}2.29(0.46 \\
11.38)\end{array}$ & $\begin{array}{l}3.58(0.71 \\
18.02)\end{array}$ & $\begin{array}{l}5.34(0.85, \\
33.71)\end{array}$ \\
\hline & & & & & & \\
\hline & $\begin{array}{l}\text { Adjusted } \\
\text { model }^{\star}\end{array}$ & Reference & $\begin{array}{l}3.12(0.83 \\
11.63)\end{array}$ & $\begin{array}{l}1.92(0.20 \\
18.54)\end{array}$ & $\begin{array}{l}5.71(1.14 \\
28.62)\end{array}$ & $\begin{array}{l}9.17(1.02, \\
82.22)\end{array}$ \\
\hline \multirow[t]{2}{*}{ EQ-5D index } & $\begin{array}{l}\text { Crude } \\
\text { model }\end{array}$ & Reference & $\begin{array}{l}-0.029(- \\
0.052,- \\
0.063)\end{array}$ & $\begin{array}{l}-0.013 \\
(-0.032 \\
0.007)\end{array}$ & $\begin{array}{l}-0.088 \\
(-0.170 \\
-0.006)\end{array}$ & $\begin{array}{l}-0.046 \\
(-0.091 \\
-0.001)\end{array}$ \\
\hline & $\begin{array}{l}\text { Adjusted } \\
\text { model }^{*}\end{array}$ & Reference & $\begin{array}{l}-0.017(- \\
0.038,0.005)\end{array}$ & $\begin{array}{l}-0.001 \\
(-0.018 \\
0.017)\end{array}$ & $\begin{array}{l}-0.026 \\
(-0.149 \\
0.018)\end{array}$ & $\begin{array}{l}-0.025 \\
(-0.072 \\
0.022)\end{array}$ \\
\hline
\end{tabular}

Data are presented as a ratio ( $95 \%$ confidence interval) or a difference estimate ( $95 \%$ confidence interval).

*Adjusted for age, sex, BMl, education (categorized as $>$ high school or $\leq$ high school), and family income (categorized as low or high). 
EQ-5D, EuroQoL five dimensions; TB, tuberculosis.

In the analyses according to severity of restrictive ventilatory disorder, subjects with mild restrictive ventilatory disorders were more likely to have any respiratory symptoms $(\mathrm{aOR}=1.95,95 \% \mathrm{Cl}=1.07-3.56)$ compared to those with normal ventilation, and these findings were specifically significant for sputum $(\mathrm{aOR}=2.28,95 \% \mathrm{Cl}=1.17-4.49)$. In comparison, moderate $(\mathrm{aOR}=9.17,95 \% \mathrm{Cl}=1.02-82.22)$ and severe $(\mathrm{aOR}=9.17,95 \% \mathrm{Cl}=1.02-82.22)$ restrictive ventilatory disorders were associated with more physical activity limitations compared to those with normal ventilation (Table 4).

\section{Discussion}

To the best of our knowledge, this is the first study to evaluate respiratory symptoms, physical activity limitations, and QoL according to type of ventilatory disorder and its severity in post-TB subjects. Among post-TB subjects, approximately $29 \%$ and $16 \%$ developed obstructive and restrictive ventilatory disorders, respectively. Severe obstructive ventilatory disorders were associated with more respiratory symptoms, more physical activity limitations, and poorer quality of life. Severe restrictive ventilatory disorder was associated with more physical activity limitations.

TB survivors frequently experience structural and functional lung sequelae that vary in severity.[7] For example, approximately $24-35 \%$ of TB survivors have been shown to experience obstructive ventilatory disorders.[19-21] In agreement with previous reports, $29 \%$ of the post-TB subjects in this study had obstructive ventilatory disorders. Thus, development of obstructive ventilatory disorders can cause important health-related burden in TB survivors. Post-TB subjects also can experience restrictive ventilatory disorders. Post-TB survivors often show a fibrotic pattern on chest imaging due to the sequelae of pulmonary TB including destruction of lung parenchyma.[22] Restrictive ventilatory disorders occur in post-TB subjects due to volume loss, lung scarring with reduction of pulmonary compliance, and an increase in elastic retraction pressure.[23, 24] In contrast to the literature on obstructive ventilatory disorders, only a few studies have described restrictive ventilatory disorders, and the prevalence was reported as $31-42 \%$ among TB survivors. $[25,26]$ The small number of patients in these studies $(n=107$ and $n=33$ ) limits the study findings. $[25,26]$ Thus, our study has the advantage of confirming these findings with the largest number of subjects using a nationwide database.

As shown in previous studies,[19, 27] clinical factors associated with poorer QoL (e.g., old age, male sex, smoking history, lower BMI, and lower education level) were more common in post-TB subjects with obstructive ventilatory disorders than in those with normal ventilation. Approximately $72 \%$ of post-TB subjects with obstructive ventilatory disorders in this study were current or ex-smokers. In line with this finding, smoking is a well-established factor associated with obstructive ventilatory disorder in post-TB subjects.[19] However, little is known as to whether development of obstructive ventilatory disorder is associated with higher symptomatic burdens in post-TB survivors, as most previous studies focused on the presence of obstructive ventilatory disorders and their severity after TB treatment.[5, 11,28] From this perspective, our study is meaningful in elucidating that development of severe obstructive ventilatory 
disorders was associated with more respiratory symptoms, more physical activity limitations, and poorer QoL compared to patients with normal ventilation; however, the findings were not significant in patients with mild-to-moderate obstructive ventilatory disorders. These results indicate that regular health checkup with pulmonary function measurement after completing TB treatment is necessary to detect obstructive ventilatory disorders early and provide appropriate treatment to prevent further lung function impairment. Recent studies also support this suggestion in showing clinical improvement after bronchodilator treatment in TB-destroyed lung patients with obstructive ventilatory disorder.[29, 30]

Despite the prevalence of restrictive ventilatory disorders after TB, to the best of our knowledge, no studies have evaluated the association between restrictive ventilatory disorder and respiratory symptoms, physical activity limitations, and QoL in TB survivors. Our study revealed that respiratory symptoms and QoL were not significantly impaired in post-TB subjects with restrictive ventilatory disorders, while physical activity limitations were significant in post-TB subjects with moderate-to-severe restrictive ventilatory disorders. Restrictive ventilatory disorders might be an underappreciated cause of functional impairments and respiratory symptoms.[31,32] One study showed that $35.4 \%$ of subjects with restrictive ventilatory disorders reported at least one chronic respiratory symptom.[32] One reason why our study results are contrary to previous findings might be that most subjects with restrictive ventilatory disorders in our study had a mild degree of restrictive abnormality; thus, the number of subjects with moderate-tosevere restrictive ventilatory was relatively small to exert statistical significance. Accumulating evidence has shown that restrictive ventilatory disorders are related to physical activity limitations, which is in line with our study results.[33, 34] The significant association of moderate-to-severe restrictive ventilatory disorders with physical activity limitations, but not with respiratory symptoms, suggests that restrictive ventilatory disorders influence physical activity limitations through mechanisms that are at least partly independent of respiratory symptoms. The decreased lung or chest wall compliance and increased elastic work of breathing might be a mechanism underlying the physical activity limitations in patients with advanced restrictive ventilatory disorders.[35]

This study has several limitations. First, this study was performed in a representative sample of Korea. Thus, our data might not be generalizable to other ethnic groups or populations. Second, obstructive ventilatory disorders were defined by pre-bronchodilator spirometric results. This might lead to an overestimate of the prevalence of obstructive ventilatory disorders. However, our estimates were similar with those of previous studies.[19] Third, the relatively small number of post-TB subjects with moderateto-severe restrictive ventilatory disorders might lead to statistical nonsignificance when analyzing the impact of ventilatory disorder severity on respiratory symptoms or QoL.

In conclusion, among the TB survivors, $29 \%$ had obstructive ventilatory disorders and $16 \%$ had restrictive ventilatory disorders. Severe obstructive ventilatory disorders were associated with increased healthrelated burden, including more respiratory symptoms, more physical activity limitations, and poorer QoL, while severe restrictive ventilatory disorder was associated with more physical activity limitations. More research is needed to establish strategies for early diagnosis and adequate treatment of ventilatory disorders in TB survivors. 


\section{Abbreviations}

TB, tuberculosis; QoL, quality of life; COPD, chronic obstructive pulmonary disease; $\mathrm{Cl}$, confidence interval; COPD, chronic obstructive pulmonary disease; NHANES, National Health and Nutrition Examination Survey; BMI, body mass index; EQ-5D, EuroQoL five dimensions; $\mathrm{FEV}_{1}$, forced expiratory volume in 1 second; FVC, forced vital capacity; OR, odd ratio.

\section{Declarations}

\section{Acknowledgments}

This work was supported by a National Research Foundation of Korea (NRF) grant funded by the Ministry of Science, Information, and Communications Technologies (MSIT) (No. 2020R1F1A1070468 and No. 2021M3E5D1A0101517621 to HL) and by the Korea Medical Device Development Fund grant funded by the Korean government (Ministry of Science and ICT, Ministry of Trade, Industry and Energy, the Ministry of Health \& Welfare, the Ministry of Food and Drug Safety) (No. KMDF202014X08-03 to HL).

\section{Authors' contributions}

H.L. and H.Y.P. had full access to all of the data in the study and take responsibility for the integrity of the data and the accuracy of the data analysis. H.L., H.Y.P., B.Y. and H.C. contributed substantially to the study design, data collection, analysis and interpretation, and the writing of the manuscript. S.H.S., Y.K. and J-Y.M .contributed substantially to the study design, data collection, and review of the manuscript. All authors read and approved the final manuscript.

\section{Funding}

This study did not receive any specific grant from funding agencies in the public, commercial, or not-forproft sectors.

\section{Availability of data and materials}

The data sets analysed during the current study are available from the corresponding author upon reasonable request.

\section{Ethics approval and consent to participate}

The study protocol was approved by the Institutional Review Board of Chungbuk National University Hospital (application no. 2021-01-041).

\section{Consent for publication}

Not applicable. 


\section{Competing interests}

None to declare.

\section{Author details}

${ }^{1}$ Division of Pulmonary and Critical Care Medicine, Department of Internal Medicine, Chungbuk National University Hospital, Chungbuk National University College of Medicine, Cheongju, Korea. ${ }^{2}$ Division of Pulmonary, Allergy, and Critical Care Medicine, Department of Internal Medicine, Hallym University Kangnam Sacred Heart Hospital, Hallym University College of Medicine, Seoul, Korea. ${ }^{3}$ Division of Pulmonary and Critical Care Medicine, Department of Medicine, Samsung Medical Center, Sungkyunkwan University School of Medicine, Seoul, Korea. ${ }^{4}$ Division of Pulmonary, Allergy, and Critical Care Medicine, Department of Internal Medicine, Hallym University Chuncheon Sacred Heart Hospital, Chuncheon, Korea. ${ }^{5}$ Lung Research Institute, Hallym University College of Medicine, Chuncheon, Korea. ${ }^{6}$ Division of Pulmonary Medicine and Allergy, Department of Internal Medicine, Hanyang University College of Medicine, Seoul, Korea

\section{References}

1. Organization WH: Global tuberculosis report 2020: executive summary. 2020.

2. Meghji J, Lesosky M, Joekes E, Banda P, Rylance J, Gordon S, Jacob J, Zonderland H, MacPherson P, Corbett EL, et al. Patient outcomes associated with post-tuberculosis lung damage in Malawi: a prospective cohort study. Thorax. 2020;75:269-78.

3. Hnizdo E, Singh T, Churchyard G. Chronic pulmonary function impairment caused by initial and recurrent pulmonary tuberculosis following treatment. Thorax. 2000;55:32-8.

4. Plit ML, Anderson R, Van Rensburg CE, Page-Shipp L, Blott JA, Fresen JL, Feldman C. Influence of antimicrobial chemotherapy on spirometric parameters and pro-inflammatory indices in severe pulmonary tuberculosis. Eur Respir J. 1998;12:351-6.

5. Ross J, Ehrlich RI, Hnizdo E, White N, Churchyard GJ. Excess lung function decline in gold miners following pulmonary tuberculosis. Thorax. 2010;65:1010-5.

6. Maguire GP, Anstey NM, Ardian M, Waramori G, Tjitra E, Kenangalem E, Handojo T, Kelly PM. Pulmonary tuberculosis, impaired lung function, disability and quality of life in a high-burden setting. Int J Tuberc Lung Dis. 2009;13:1500-6.

7. Pasipanodya JG, McNabb SJ, Hilsenrath P, Bae S, Lykens K, Vecino E, Munguia G, Miller TL, Drewyer G, Weis SE. Pulmonary impairment after tuberculosis and its contribution to TB burden. BMC Public Health. 2010;10:259.

8. Ravimohan S, Kornfeld H, Weissman D, Bisson GP. Tuberculosis and lung damage: from epidemiology to pathophysiology. Eur Respir Rev 2018, 27. 
9. Rhee CK, Yoo KH, Lee JH, Park MJ, Kim WJ, Park YB, Hwang YI, Kim YS, Jung JY, Moon JY, et al. Clinical characteristics of patients with tuberculosis-destroyed lung. Int J Tuberc Lung Dis. 2013;17:67-75.

10. Ralph AP, Kenangalem E, Waramori G, Pontororing GJ, Sandjaja, Tjitra E, Maguire GP, Kelly PM, Anstey NM. High morbidity during treatment and residual pulmonary disability in pulmonary tuberculosis: under-recognised phenomena. PLoS One. 2013;8:e80302.

11. Ehrlich RI, Adams S, Baatjies R, Jeebhay MF. Chronic airflow obstruction and respiratory symptoms following tuberculosis: a review of South African studies. Int J Tuberc Lung Dis. 2011;15:886-91.

12. Manji M, Shayo G, Mamuya S, Mpembeni R, Jusabani A, Mugusi F. Lung functions among patients with pulmonary tuberculosis in Dar es Salaam - a cross-sectional study. BMC Pulm Med. 2016;16:58.

13. Miller MR, Hankinson J, Brusasco V, Burgos F, Casaburi R, Coates A, Crapo R, Enright Pv, Van der Grinten C, Gustafsson P. Standardisation of spirometry. European respiratory journal. 2005;26:31938.

14. Choi JK, Paek D, Lee JO. Normal predictive values of spirometry in Korean population. Tuberculosis Respiratory Diseases. 2005;58:230-42.

15. Lee H, Shin SH, Gu S, Zhao D, Kang D, Joi YR, Suh GY, Pastor-Barriuso R, Guallar E, Cho J, Park HY. Racial differences in comorbidity profile among patients with chronic obstructive pulmonary disease. BMC Med. 2018;16:178.

16. Lange P, Celli B, Agustí A, Boje Jensen G, Divo M, Faner R, Guerra S, Marott JL, Martinez FD, MartinezCamblor P, et al. Lung-Function Trajectories Leading to Chronic Obstructive Pulmonary Disease. N Engl J Med. 2015;373:111-22.

17. Rabe KF, Hurd S, Anzueto A, Barnes PJ, Buist SA, Calverley P, Fukuchi Y, Jenkins C, Rodriguez-Roisin $\mathrm{R}$, van Weel C, Zielinski J. Global strategy for the diagnosis, management, and prevention of chronic obstructive pulmonary disease: GOLD executive summary. Am J Respir Crit Care Med. 2007;176:532-55.

18. Sim YS, Lee JH, Lee WY, Suh DI, Oh YM, Yoon JS, Lee JH, Cho JH, Kwon CS, Chang JH. Spirometry and Bronchodilator Test. Tuberc Respir Dis (Seoul). 2017;80:105-12.

19. Jung JW, Choi JC, Shin JW, Kim JY, Choi BW, Park IW. Pulmonary Impairment in Tuberculosis Survivors: The Korean National Health and Nutrition Examination Survey 2008-2012. PLoS One. 2015;10:e0141230.

20. Lam KB, Jiang CQ, Jordan RE, Miller MR, Zhang WS, Cheng KK, Lam TH, Adab P. Prior TB, smoking, and airflow obstruction: a cross-sectional analysis of the Guangzhou Biobank Cohort Study. Chest. 2010;137:593-600.

21. Snider GL, Doctor L, Demas TA, Shaw AR. Obstructive airway disease in patients with treated pulmonary tuberculosis. Am Rev Respir Dis. 1971;103:625-40.

22. Pasipanodya JG, Vecino E, Miller TL, Munguia G, Drewyer G, Fernandez M, Slocum P, Weis SE. Nonhispanic whites have higher risk for pulmonary impairment from pulmonary tuberculosis. BMC Public Health. 2012;12:119. 
23. Di Naso FC, Pereira JS, Schuh SJ, Unis G. [Functional evaluation in patients with pulmonary tuberculosis sequelae]. Rev Port Pneumol. 2011;17:216-21.

24. Miguel-Reyes JL, Gochicoa-Rangel L, Pérez-Padilla R, Torre-Bouscoulet L. Functional respiratory assessment in interstitial lung disease. Rev Invest Clin. 2015;67:5-14.

25. Pasipanodya JG, Miller TL, Vecino M, Munguia G, Garmon R, Bae S, Drewyer G, Weis SE. Pulmonary impairment after tuberculosis. Chest. 2007;131:1817-24.

26. de Vallière $S$, Barker RD. Residual lung damage after completion of treatment for multidrug-resistant tuberculosis. Int J Tuberc Lung Dis. 2004;8:767-71.

27. Gandhi K, Gupta S, Singla R. Risk factors associated with development of pulmonary impairment after tuberculosis. Indian J Tuberc. 2016;63:34-8.

28. Willcox PA, Ferguson AD. Chronic obstructive airways disease following treated pulmonary tuberculosis. Respir Med. 1989;83:195-8.

29. Kim C-J, Yoon H-K, Park M-J, Yoo K-H, Jung K-S, Park J-W, Lim SY, Shim JJ, Lee YC, Kim Y-S, et al. Inhaled indacaterol for the treatment of COPD patients with destroyed lung by tuberculosis and moderate-to-severe airflow limitation: results from the randomized INFINITY study. Int J Chronic Obstr Pulm Dis. 2017;12:1589-96.

30. Kim HC, Kim TH, Kim Y-J, Rhee CK, Oh Y-M. Effect of tiotropium inhaler use on mortality in patients with tuberculous destroyed lung: based on linkage between hospital and nationwide health insurance claims data in South Korea. Respiratory research. 2019;20:85-5.

31. Godfrey MS, Jankowich MD. The Vital Capacity Is Vital: Epidemiology and Clinical Significance of the Restrictive Spirometry Pattern. Chest. 2016;149:238-51.

32. Mannino DM, Doherty DE, Sonia Buist A. Global Initiative on Obstructive Lung Disease (GOLD) classification of lung disease and mortality: findings from the Atherosclerosis Risk in Communities (ARIC) study. Respir Med. 2006;100:115-22.

33. Chung SJ, Kim HI, Yang B, Kim T, Sim YS, Kang HK, Kim SH, Yoon HJ, Choi H, Lee H. Impact of the severity of restrictive spirometric pattern on nutrition, physical activity, and quality of life: results from a nationally representative database. Sci Rep. 2020;10:19672.

34. Guerra S, Sherrill DL, Venker C, Ceccato CM, Halonen M, Martinez FD. Morbidity and mortality associated with the restrictive spirometric pattern: a longitudinal study. Thorax. 2010;65:499-504.

35. Nonato NL, Nascimento OA, Padilla RP, de Oca MM, Tálamo C, Valdivia G, Lisboa C, López MV, Celli $B$, Menezes AM, Jardim JR. Occurrence of respiratory symptoms in persons with restrictive ventilatory impairment compared with persons with chronic obstructive pulmonary disease: The PLATINO study. Chron Respir Dis. 2015;12:264-73.

\section{Figures}




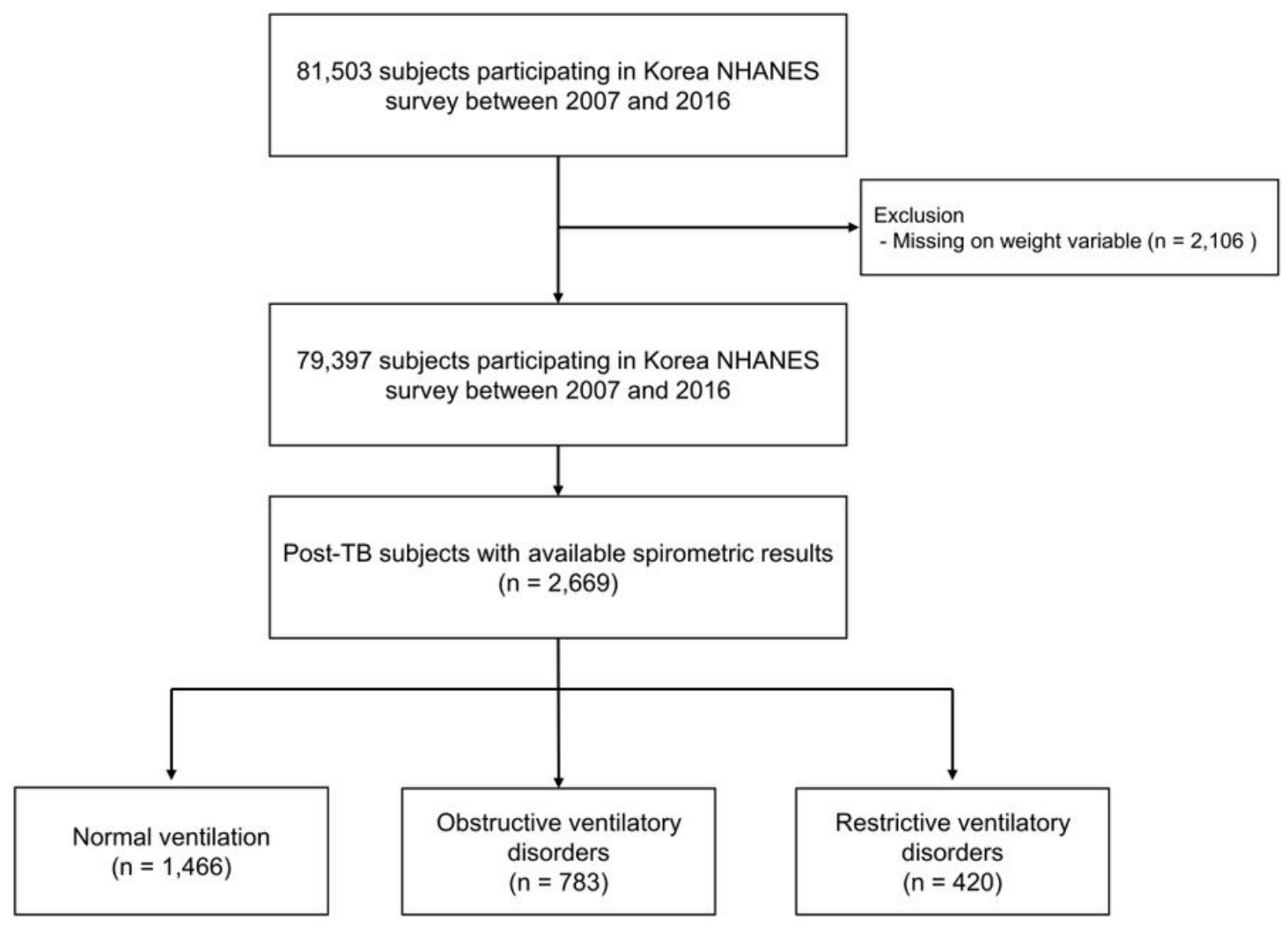

\section{Figure 1}

Flow chart of patient selection TB, Tuberculosis; NHNES, National Health and Nutrition Examination Survey. 